\title{
Pair-Distribution Functions of Laughlin Quasielectrons in Partially Filled Composite Fermion Levels
}

\author{
D. Wodziński ${ }^{a}, \mathrm{~A}$. Wóss ${ }^{a, b}$ AND J.J. QUINN ${ }^{b}$ \\ ${ }^{a}$ Institute of Physics, Wrocław University of Technology \\ Wybrzeże Wyspiańskiego 27, 50-370 Wrocław, Poland \\ ${ }^{b}$ University of Tennessee, Knoxville, Tennessee 37996, USA
}

Pair-distribution functions $g(r)$ of the Laughlin quasielectrons are calculated in the fractional quantum Hall states at electron filling factors $\nu=4 / 11$ and $3 / 8$. They all have a shoulder at a medium range, supporting the idea of quasielectron cluster formation. The intra- and inter-cluster contributions to $g(r)$ are identified. The average cluster sizes are estimated; pairs and triplets of quasielectrons are suggested at $\nu=4 / 11$ and $3 / 8$, respectively.

PACS numbers: 71.10.Pm, 73.43.-f

\section{Introduction}

Pan et al. [1] have recently observed fractional quantum Hall effect (FQHE) in a spin-polarized two-dimensional electron gas (2DEG) at electron filling factors $\nu=\frac{4}{11}, \frac{3}{8}$, and $\frac{5}{13}$. These values correspond to $\nu_{\mathrm{QE}}=\frac{1}{3}, \frac{1}{2}$, and $\frac{2}{3}$ of the Laughlin quasielectrons (QE's), respectively. In the composite fermion (CF) model [2], each QE corresponds to a CF in the second Landau level (LL). Pan's discovery implies that the QE's or CF's can also form incompressible states when partially filling a shell. This could not be predicted by a simple analogy with known fractional electron liquids (Laughlin [3], Jain [2], or Moore-Read [4] states), because of a different form of the QE-QE interaction [5].

From exact numerical diagonalization on a Haldane sphere [6], we have obtained the energy spectra and wave functions of up to 14 interacting QE's. We have identified the series of finite-size liquid ground states with a gap, which extrapolate to the experimentally observed incompressible $\mathrm{FQH}$ states. In these 
states, we have calculated QE-QE pair-distribution functions $g(r)$, and showed that they increase as $\sim r^{2}$ at short range and have a pronounced shoulder at a medium range. This behavior supports the idea of QE cluster formation, suggested earlier [7] from the analysis of QE-QE interaction pseudopotential. The $g(r)$ is decomposed into short- and long-range contributions, interpreted as correlations between the QE's from the same or different clusters. The inter-cluster QE-QE correlations appear to be the same in all three $\nu_{\mathrm{QE}}=\frac{1}{3}, \frac{1}{2}$, and $\frac{2}{3}$ states. The cluster size on the other hand does depend on $\nu$, and we argue that the QE's form pairs at $\nu_{\mathrm{QE}}=\frac{1}{3}$ and triplets at $\nu_{\mathrm{QE}}=\frac{1}{2}$.

\section{Numerical calculations}

To investigate the system we use Haldane's idea [6] of putting $N$ particles of charge $q$ on a spherical surface of radius $R$. Dirac monopole of strength $2 Q$ placed in the center of the sphere is the source of magnetic field $B$, and $2 Q \phi_{0}=4 \pi R^{2} B$. Here $\phi_{0}=h c / q$ is the elementary flux. Using the definition of the magnetic length, $\lambda=\sqrt{\hbar c / q B}$, this can be written as $Q \lambda^{2}=R^{2}$. In the following, $\lambda$ denotes the $\mathrm{QE}$ magnetic length corresponding to the fractional charge $q=-e / 3$.

Each particle on the lowest LL has angular momentum $l=Q$ and its $z$-component $m$ can take on values from $-l$ to $l$ so degeneracy of the shell is equal to $\Gamma=2 l+1$. The single-particle configurations $\left|m_{1}, m_{2}, \ldots, m_{N}\right\rangle$ can be chosen as a basis of the Hilbert space. Diagonalizing the matrix of interaction Hamiltonian we obtain energy as a function of total angular momentum $L$. Since number of electrons required to represent FQHE at $\nu=\frac{3}{8}, \frac{4}{11}$, and $\frac{5}{13}$ is too large to be diagonalized exactly, we neglect quasiparticles on the lowest CF-LL and take into account only CF's from the second, partially filled CF-LL. Interaction between these particles is given by QE-QE pseudopotential $V(\mathcal{R})$, where the relative pair angular momentum $\mathcal{R}=2 l-L$ increases with average pair separation $\sqrt{\left\langle r^{2}\right\rangle}$.
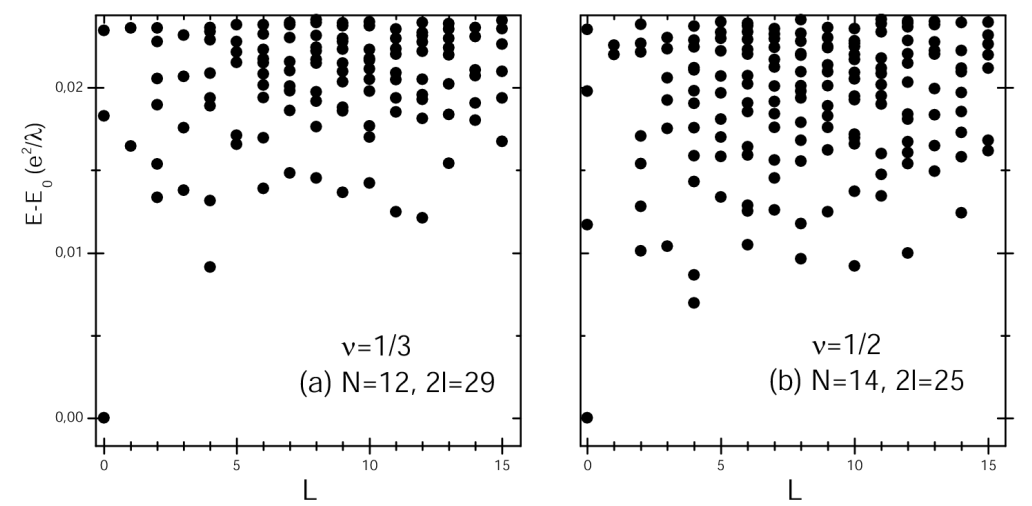

Fig. 1. Excitation energy spectra ( $E_{0}$ is the ground state energy) of $N$ interacting QE's on a sphere, at the values of CF-LL degeneracy $\Gamma=2 l+1$ corresponding to the incompressible ground states at the QE filling factors $\frac{\nu=1}{3}$ (a) and $\frac{1}{2}$ (b). 
Showing small value at $\mathcal{R}=1$ and strong maximum at $\mathcal{R}=3[5,8,9]$, this pseudopotential differs from a superlinearly decreasing one, describing the Coulomb interaction among electrons. This difference precludes the Laughlin correlations among QE's.

Incompressible states are represented by these combinations of particles number $N$ and shell degeneracy $\Gamma$ for which ground state is significantly separated from excited states. For the Laughlin liquid it occurs for $2 l=3 N-3$. In contrast, for QE such situation takes place for sequence $2 l=3 N-7$ (Fig. 1) representing $\nu_{\mathrm{QE}}=\frac{1}{3} \approx N / \Gamma$ and for its particle-hole conjugates series (i.e. sequences with $N$ replaced by $\Gamma-N) 2 l=\frac{3}{2} N+2\left(\nu=\frac{2}{3}\right)[7]$.

Another sequence was anticipated at $2 l=2 N+1$ and $2 l=2 N-3$ to represent the infinite $\nu=\frac{3}{8} \mathrm{FQH}$ state. Unfortunately, we are unable to compute the spectra for $N \geq 18$ and it seems that in this case incompressible states occur only for odd values of $\frac{1}{2} N$. Hence we have only two configurations for each sequence. Nevertheless, we expect that the ground state for $N=14$ and $2 l=25$ (and its particle-hole counterpart at $N=12$ and the same $2 l=25$ ) may possibly represent the $\nu=\frac{3}{8}$ FQH state (i.e., have similar correlations causing incompressibility).

\section{Pair-distribution function}

Pair-distribution functions $g(r)$ were calculated as expectation value of operator $\hat{g}(r)=(2 / N)^{2} \delta(R \theta-r)$ for previously found wave function of non-degenerate ground state of QE system. Here, $\theta$ is the relative angle on a sphere and $r$ measures interparticle distance along the sphere surface. Denoting infinitesimal area by $\mathrm{d} S=2 \pi R^{2} \mathrm{~d}(\cos \theta)$ or (in magnetic units) by $\mathrm{d} s=\mathrm{d} S / 2 \pi \lambda^{2}$, we get a normalization condition in large systems

$$
\int[1-g(r)] \mathrm{d} s=\frac{2 l}{N} \rightarrow \nu^{-1} .
$$

Since $\mathrm{d} s=l \mathrm{~d}(\cos \theta)$, a "local filling factor" can also be defined as $\nu(r)=\mathrm{d} N / \mathrm{d} s=$ $(N / 2 l) g(r)$, and it satisfies $\nu(\infty)=\nu$ and $\int \nu(r) \mathrm{d} s=N-1$.

For a full lowest LL $\left(\nu_{\mathrm{e}}=1\right)$ the pair-distribution function is [10]:

$$
g_{0}=1-\exp \left(-r^{2} / 2 \lambda^{2}\right) \text {. }
$$

For the sequence $2 l=3 N-7$, representing $\nu_{\mathrm{QE}}=\frac{1}{3}$ state, the behavior of $g(r)$ looks like in Fig. 1a. For small $r$ it is similar to $g_{0}$, i.e., $g(r) \propto r^{2}$, but the curve bends around $r=2.5 \lambda$. The shoulder appears for all calculated $N$, and evidently it is not an effect of a finite number of particles. Similar shoulders occur also in $g(r)$ for $\nu_{\mathrm{QE}}=\frac{2}{3}$, i.e. $\nu=\frac{5}{13}$ state. In Fig. $2 \mathrm{~b}$ we have compared this curve with pair-correlation functions obtained for other incompressible FQH states (full LL, Laughlin $\nu=\frac{1}{3}$ state, or Moore-Read half-filled state). Qualitative difference between these functions indicates that correlations responsible for the FQHE at new $\nu=\frac{4}{11}$ and $\frac{3}{8}$ fillings states cannot be the same. 


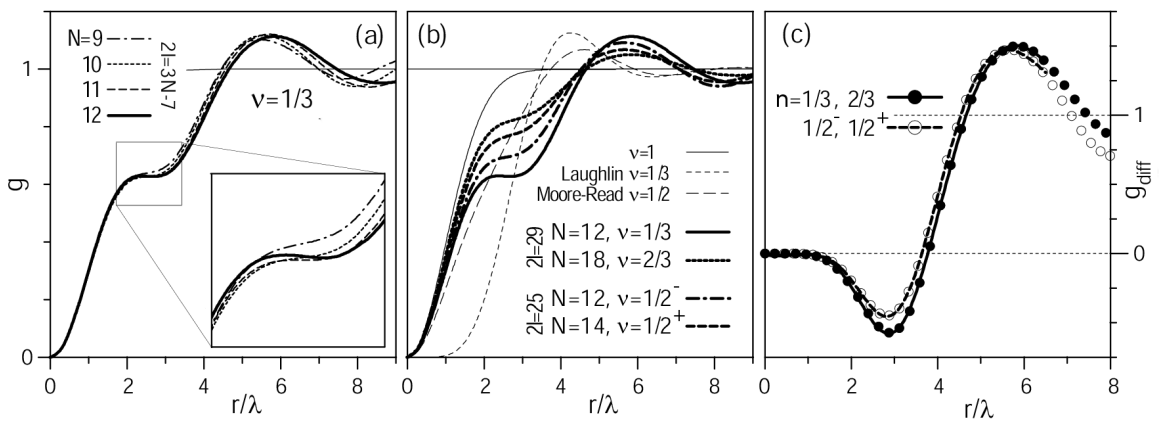

Fig. 2. QE-QE pair-distribution functions $g(r)$ of the incompressible ground states at different QE filling factors $\nu$. (a) Curves for $\nu=\frac{1}{3}$ and different QE numbers $N$; inset - blowup of the plateau region; (b) curves for QE's at different $\nu$ (thick lines) compared to some known incompressible states of electrons; (c) the "remainder" $g_{\text {diff }}(r)$ defined by Eq. (3).

The shoulder in $g(r)$ may result from different correlation between QE's close to each other and between distant ones, consistently with an idea of clusters formation $[7,11,12]$. To find inter- and intra-cluster correlation we decompose $g(r)$ into $g_{0}$ describing a full lowest LL and a (properly normalized) "remainder" $g_{\text {diff }}$ :

$$
g(r)=\alpha g_{0}(r)+(1-\alpha) g_{\text {diff }}(r) .
$$

Parameter $\alpha$ is calculated as the limit of $g / g_{0}$ at $r \rightarrow 0$. The curves $g_{\text {diff }}$ obtained for $\nu_{\mathrm{QE}}=\frac{1}{3}$ and $\frac{1}{2}$ are very similar and for the pairs of particle-hole conjugate states they are identical (Fig. 2c). The vanishing of $g_{\text {diff }}(r)$ at short-range reflects isolation of QE's belonging to different clusters. Negative value around $r=3$ indicates that intra-cluster correlations are accurately described by $g_{0}$ only within a certain radius.

Similar form of $g(r)$ was found [13] for broken-symmetry Laughlin states, in which the shoulder results from angular averaging of an anisotropic function $g(r, \phi) \sim r^{2}$ or $r^{6}$, depending on $\phi$. However, the present case of QE's is different, because $g(r)$ is isotropic (nondegenerate ground state wave functions have $L=0$ ) and the shoulders result from radial averaging of inter- and intra-cluster correlations (beginning as $\sim r^{2}$ and a higher power of $r$ at short range, respectively).

\section{Average cluster size}

Let us assume that our system consists of independent clusters, each with $K$ particle. By independence of the clusters we mean that inter-cluster correlations do not affect the local filling factor $\nu(r)$ at short range. Such system for small $r$ should have $\nu(r)$ similar to $\nu_{K}(r)$ of a single $K$-cluster. For that cluster, which on a sphere is the $K$-particle state with the maximum total angular momentum 
$L=K l-\frac{1}{2} K(K-1)$, we have calculated $g_{K}(r)$ and then by taking $\nu_{K}(r)=$ $\nu g_{K}(r) \approx \beta_{K} g_{0}(r)$ we found prefactor $\beta_{K}$ for different values of $K$ and $2 l$. Some results are listed in Table. Now we can compare these values $\beta_{K}$ with $\beta=(N / 2 l) \alpha$ obtained for our investigated incompressible $N$-QE systems.

\section{TABLE}

Parameters $\beta_{K}$ of the short-range approximation $\nu(r) \sim \beta g_{0}(r)$ obtained for independent clusters of size $K$.

\begin{tabular}{c|c|c|c|c|c}
\hline \hline $2 l$ & $\beta_{2}$ & $\beta_{3}$ & $\beta_{4}$ & $\beta_{5}$ & $\beta_{6}$ \\
\hline 25 & 0.2768 & 0.4196 & 0.5110 & 0.5765 & 0.6269 \\
29 & 0.2730 & 0.4134 & 0.5029 & 0.5669 & 0.6159 \\
60 & 0.2609 & 0.3938 & 0.4778 & 0.5372 & 0.5821 \\
$\infty$ & 0.2500 & 0.3763 & 0.4555 & 0.5110 & 0.5527
\end{tabular}

Obviously, assumption that our clusters are independent is only approximation. Apart from that, we know that $\nu_{\mathrm{QE}}=\frac{1}{3}$ states occur for all $N$ (not only divisible by two or three) so different clusters could have different sizes $K$. Also, parameters $\alpha$ and $\beta$ are size-dependent and their extrapolation to large systems is not very reliable based on limited number of $N$-QE systems we are able to diagonalize. Nevertheless, we can test the method using the Moore-Read state known to be paired [4, 14]. Our calculation for $N=14$ and $2 l=25$ gives $\beta_{\mathrm{MR}}=0.336$, somewhat larger than $\beta_{2}$. Hence, we shall assume that $\beta_{K}$ in general underestimates the actual value of $\beta$ in a many-body $K$-clustered state.

For the $\mathrm{QE}$ system at $\nu_{\mathrm{QE}}=\frac{1}{3}(N=12$ and $2 l=29)$, we got $\beta=$ $0.319 \approx \beta_{\mathrm{MR}}$, and $\beta=0.479$ for $\nu_{\mathrm{QE}}=\frac{1}{2}^{+}(N=14$ and $2 l=25)$. In the light of these facts it seems probable that QE's (on the average) form pairs at $\nu_{\mathrm{QE}}=\frac{1}{3}$ and triplets at $\nu_{\mathrm{QE}}=\frac{1}{2}$.

\section{Conclusion}

Pair-distribution functions of new FQH states differ significantly from these known for electrons at $\nu=1, \frac{1}{3}$ (Laughlin), or $\frac{1}{2}$ (Moore-Read). For small $r$ they behave like $g(r)$ for electrons occupying completely the lowest LL, and then for $r \approx 2.5 \lambda$ they have a shoulder. Our results support hypothesis that QE's form clusters. Short- and long-range contribution to $g(r)$, describing correlations between the QE's from the same and different clusters, have been found. Both correlations depend rather weakly on $\nu$. We also estimated average size of the clusters, which seem to form pairs at $\nu_{\mathrm{QE}}=\frac{1}{3}$ and triplets at $\nu_{\mathrm{QE}}=\frac{1}{2}$. 


\section{Acknowledgments}

The authors thank W. Pan, W. Bardyszewski, and L. Bryja for helpful discussions. This work was supported by grant DE-FG 02-97ER45657 of the Materials Science Program - Basic Energy Sciences of the U.S. Dept. of Energy and grant 2P03B02424 of the State Committee for Scientific Research (Poland).

\section{References}

[1] W. Pan, H.L. Störmer, D.C. Tsui, L.N. Pfeiffer, K.W. Baldwin, K.W. West, Phys. Rev. Lett. 90, 016801 (2003); conjugate $\nu=7 / 11$ state was first observed by V.J. Goldman, M. Shayegan, Surf. Sci. 229, 10 (1990).

[2] J.K. Jain, Phys. Rev. Lett. 63, 199 (1989).

[3] R.B. Laughlin, Phys. Rev. Lett. 50, 1395 (1983).

[4] G. Moore, N. Read, Nucl. Phys. B 360, 362 (1991).

[5] A. Wójs, J.J. Quinn, Phys. Rev. B 61, 2846 (2000).

[6] F.D.M. Haldane, Phys. Rev. Lett. 51, 605 (1983).

[7] A. Wójs, K.-S. Yi, J.J. Quinn, Phys. Rev. B 69, 205322 (2004).

[8] P. Sitko, S.N. Yi, K.-S. Yi, J.J. Quinn, Phys. Rev. Lett. 76, 3396 (1996).

[9] S.-Y. Lee, V.W. Scarola, J.K. Jain, Phys. Rev. Lett. 87, 256803 (2001); Phys. Rev. B 66, 085336 (2002).

[10] B. Jancovici, Phys. Rev. Lett. 46, 386 (1981).

[11] M. Flohr, K. Osterloh, Phys. Rev. B 67, 235316 (2003).

[12] A. Wójs, K.-S. Yi, J.J. Quinn, Acta Phys. Pol. A 103, 517 (2003); J.J. Quinn, A. Wójs, K.-S. Yi, Phys. Lett. A 318, 152 (2003).

[13] K. Musaelian, R. Joynt, J. Phys., Condens. Matter 8, L105 (1996); O. Ciftja, C. Wexler, Phys. Rev. B 65, 045306 (2002); Phys. Rev. B 65, 205307 (2002).

[14] A. Wójs, J.J. Quinn, Phys. Rev. B 71, 045324 (2005). 